\title{
Identification of Urothelial Cancer-Associated 1 (UCA1) as a diagnostic biomarker of pre-eclampsia via regulating microRNA-16 and its downstream signaling pathway
}

\author{
Type \\ Research paper \\ Keywords \\ miR-16, UCA1, Pre-eclampsia (PE), PAPPA
}

\begin{abstract}
Introduction

In this study, we studied the clinical value of using urothelial cancer-associated 1 (UCA1) and microRNA-16 (miR-16) as biomarkers for the diagnosis of pre-eclampsia (PE). Also, we compared the diagnostic values of miR-16, UCA1 and pregnancy-associated plasma protein-A (PAPP-A) in PE. Furthermore, we investigated the interaction between miR-16 and UCA1/PAPP-A.
\end{abstract}

\section{Material and methods}

$128 \mathrm{PE}$ patients and 172 healthy pregnant women were enrolled in this study. Receiver operating characteristic (ROC) analysis was carried out to predict the diagnostic values of UCA1, miR-16 and PAPP-A in PE. Enzyme-linked immunosorbent assay (ELISA), real-time polymerase chain reaction (PCR), Western blot analysis, immunohistochemistry (IHC) assay, computational analysis, and luciferase assay were conducted to measure the differential expression of UCA1, miR-16, and PAPPA while establishing a signaling pathway of UCA1/miR-16/PAPP-A.

\section{Results}

Compared with miR-16 and PAPP-A, UCA1 exhibited a better value in the diagnosis of PE. The expression of PAPP-A and UCA1 was down-regulated while the expression of miR-16 was upregulated in patients with $\mathrm{PE}$, especially in patients with HELLP pregnancies. Moreover, UCA1 was identified as a sponge of miR-16, while PAPP-A mRNA was identified as a virtual target gene of miR-16. Finally, a negative regulatory relationship was observed between the expression of miR-16 and UCA1 or PAPP-A, while the expression of UCA1 and PAPP-A were positively related.

\section{Conclusions}

Taken together, UCA1 could be used as a more valuable biomarker in the diagnosis of PE. Meanwhile, the reduced expression of UCA1 could exert a positive effect by reducing the expression of PAPP-A in the pathogenesis of PE. 
1 Identification of Urothelial Cancer-Associated 1 (UCA1) as a diagnostic biomarker of

2 pre-eclampsia via regulating microRNA-16 and its downstream signaling pathway

3 Ye-hong Xv ${ }^{1}$, Wei Gao², Liao-jv Wang ${ }^{2 *}$

4 1. Department of Obstetrics, Northwest Women's and Children's Hospital, Xi'an, $5 \quad$ Shaanxi, China 710061

6 2. Department of Obstetrics, The First People's Hospital of Xianyang City, Xianyang, 7 Shaanxi, China 712000

$8 \quad *$ Corresponding author: Liao-jv Wang

9 Institution: Department of Obstetrics, The First People's Hospital of Xianyang City,

10 Xianyang, Shaanxi, China 712000

11 Address: No.10 Biyuan Rd, Xianyang, Shaanxi, China 712000

12 Email: PECXYH@yeah.net

13 Abstract

14 Background: In this study, we studied the clinical value of using urothelial cancer15 associated 1 (UCA1) and microRNA-16 (miR-16) as biomarkers for the diagnosis of pre16 eclampsia (PE). Also, we compared the diagnostic values of miR-16, UCA1 and pregnancy17 associated plasma protein-A (PAPP-A) in PE. Furthermore, we investigated the interaction 18 between miR-16 and UCA1/PAPP-A. Method: 128 PE patients and 172 healthy pregnant 19 women were enrolled in this study. Receiver operating characteristic (ROC) analysis was 20 carried out to predict the diagnostic values of UCA1, miR-16 and PAPP-A in PE. Enzyme21 linked immunosorbent assay (ELISA), real-time polymerase chain reaction (PCR), Western 22 blot analysis, immunohistochemistry (IHC) assay, computational analysis, and luciferase 23 assay were conducted to measure the differential expression of UCA1, miR-16, and PAPP24 A while establishing a signaling pathway of UCA1/miR-16/PAPP-A. Results: Compared 25 with miR-16 and PAPP-A, UCA1 exhibited a better value in the diagnosis of PE. The 26 expression of PAPP-A and UCA1 was down-regulated while the expression of miR-16 was 27 up-regulated in patients with PE, especially in patients with HELLP pregnancies. Moreover, 28 UCA1 was identified as a sponge of miR-16, while PAPP-A mRNA was identified as a virtual 
target gene of miR-16. Finally, a negative regulatory relationship was observed between the expression of miR-16 and UCA1 or PAPP-A, while the expression of UCA1 and PAPP-A were positively related. Conclusion: Taken together, UCA1 could be used as a more valuable biomarker in the diagnosis of PE. Meanwhile, the reduced expression of UCA1 could exert a positive effect by reducing the expression of PAPP-A in the pathogenesis of PE.

\section{Running title: MiR-16, UCA1, and PAPP-A value in PE diagnosis}

36 Keywords: Pre-eclampsia (PE), UCA1, miR-16, PAPPA

\section{Introduction}

As a complication of pregnancy, pre-eclampsia (PE) is featured by proteinuria and increased blood pressure [1]. In Western countries, the incidence of PE is about 5\%, although the incidence of PE is much higher in developing countries [2]. Worldwide, PE is attributed to over one hundred thousand maternal deaths every year and is considered as a key reason for premature delivery, i.e., children born with $<1500 \mathrm{~g}$ of birth weight [1, $3,4]$. Moreover, PE has been shown to cause cardiovascular morbidity in pregnant women $44[5]$.

Initially isolated from the serum of pregnant women, pregnancy-associated plasma 46 protein-A (PAPP-A) contains a unique metalloproteinase zinc-binding motif in its amino 47 acid structure, although the role of this motif remains unclear. PAPP-A is increasingly used 48 for the prenatal screening of trisomy 21, either alone or in combination with ultrasound measurement of nuchal translucency (NT) $[6,7]$, while recently, a study showed that 50 PAPP-A is associated with a proteolytic activity [8-11]. In fact, an abnormally low level of 51 PAPP-A has been used as a biomarker to predict the tendency to develop small-for52 gestational-age (SGA) [12]. Also, it was shown that the ultrasound examination of the 53 uterine artery can provide useful information to predict the onset of PE even in the first 54 trimester [13]. Currently, it is believed that an abnormally low level of PAPP-A plays a role 55 as essential as that of abnormal ultrasound results in the prediction of fetuses with SGA, 56 although the role of a low level of PAPP-A in the prediction of PE remains controversial 
57 [12]. These discrepancies may be due to the different molecular mechanisms implicated 58 in the development of SGA and PE, such that an abnormal ultrasound result may indicate 59 the invasion of trophoblasts in maternal spiral arteries [14], whereas a low value of PAPP60 A may indicate that the placenta can no longer support the further growth of the fetus.

61 The expression of transcriptome is regulated by a complex network involving the 62 interaction among a wide range of molecules, including messenger RNA (mRNA), 63 microRNA (miRNA), and long non-coding RNA (IncRNA) [15]. Among them, miRNA plays 64 an essential role to maintain the stability of transcriptome and to regulate the 65 homeostasis in the body. Recently, the molecular interaction between IncRNA/miRNA 66 and mRNAs has gained special interests because it has been revealed that both miRNAs, 67 a type of small non-coding RNAs (ncRNAs) with a length of about 22 nucleotides, and 68 IncRNAs, a type of ncRNAs with a length of over 200 nucleotides, are involved in the 69 translation regulation of their target mRNAs $[16,17]$. At the same time, mRNAs were also 70 shown to regulate the expression of ncRNAs in a certain way $[18,19]$.

71 In a previous study, it was found that increased expression of urothelial cancer-associated 721 (UCA1) was positively associated with the increase in imatinib (IM) resistance 73 [20]. Further investigation showed that UCA1 competitively binds to miR-16 and 74 suppressing the function of miR-16. In another study, it was demonstrated that UCA1 75 functions as competing endogenous (ceRNA) of miR-16 [20]. In bladder cancer cells, UCA1 76 could also function as an endogenous sponge of miR-16 to regulate GLS2 expression and 77 glutamine metabolism [21].

78 It has been shown that PAPP-A could function as a biomarker in the diagnosis of PE [22]. 79 Meanwhile, using an online miRNA database, we found that miR-16 might act as a 80 potential regulator of PAPP-A. Furthermore, UCA1 has been identified as a competing 81 endogenous RNA (ceRNA) of miR-16 [21]. In this study, we studied the value of using UCA1 82 and miR-16 as biomarkers in the diagnosis of PE. In addition, we compared the diagnostic 83 values of miR-16, UCA1 and PAPP-A in PE.

84 Materials and Methods 


\section{Human subjects and sample collection}

86 In this study, PE patients $(\mathrm{N}=128)$ and healthy pregnant women $(\mathrm{N}=172)$ were enrolled in the case group and the control group, respectively. Subsequently, according to the type pregnancies, which was a complication of pregnancy characterized by hemolysis, elevated liver enzymes, and a low platelet count. $\mathrm{N}=8$ ). Demographic and clinical characteristics of all subjects were collected and analyzed and demonstrated in Table 1. Moreover, peripheral blood samples and placenta samples were collected from all subjects for subsequent receiver operating characteristic (ROC) analyses. All patients have signed the written informed consent before their participation in this study. In addition, this study was approved by the Ethics Committee of our institute and was carried out in accordance with the Helsinki declaration.

\section{RNA isolation and real-time PCR}

A miRNeasy Mini Kit (Qiagen, Hilden, Germany) was used to extract the total RNA from tissue and cell samples. Isolated RNA was reversely transcribed into complementary DNA (cDNA) templates using a reverse transcription kit (Thermo Fisher Scientific, Waltham, MA). The expression of UCA1, miR-16, and PAPP-A mRNA was measured using quantitative real-time PCR and an SYBR Premix Ex Taq II reagent kit (Takara, Tokyo, Japan) following the instruction of the kit. The reaction system of quantitative real-time PCR contained a volume of $20 \mu \mathrm{l}$, including $10.0 \mu \mathrm{l}$ of One Step SYBR RT-PCR Buffer III, $0.4 \mu \mathrm{l}$ of TaKaRa Ex Taq HS, $0.4 \mu$ l of Prime Script RT Enzyme Mix $\amalg, 0.3 \mu$ l of PCR forward primer, $0.3 \mu \mathrm{l}$ of PCR reverse primer, $0.4 \mu \mathrm{l}$ of ROX Reference Dye (50x), $2 \mu \mathrm{l}$ of total RNA, and $6.2 \mu$ l of RNase Free dd $\mathrm{H}_{2} \mathrm{O}$. An ABI7500 quantitative PCR instrument (ABI, Foster City, CA) was used to carry out the quantitative real-time PCR. The reaction conditions were set as follows: pre-denaturation at $95^{\circ} \mathrm{C}$ for $10 \mathrm{~s}$, followed by 40 cycles of denaturation at $95^{\circ} \mathrm{C}$ for $5 \mathrm{~s}$ and annealing at $60^{\circ} \mathrm{C}$ for $25 \mathrm{~s}$, and a final extension step. $\mathrm{U} 6$ was used as the internal reference to quantify the relative expression of UCA1, miR-16, and PAPP-A mRNA using the $2^{-\Delta \Delta C t}$ method. Each experiment was repeated three times. 
115 Trophoblast and Hela cells were acquired from ATCC (American Type Culture Collection, 116 Manassas, VA) and maintained in an environment of saturated humidity at $37^{\circ} \mathrm{C}$ and under 5\% $\mathrm{CO}_{2}$. DMEM (Gibco, Thermo Fisher Scientific, Waltham, MA) containing 10\%

118 fetal bovine serum, 100 unit/mL streptomycin, and $100 \mathrm{mg} / \mathrm{mL}$ penicillin was used as the 119 culture medium. For transfection experiments, trophoblast and Hela cells were seeded 120 into 96-well plates at a density of $10^{4}$ cells per well and incubated overnight. On the next 121 day, the cells were transfected with pcDNA-UCA1, pcDNA-anti-miR-16 using 122 Lipofectamine 2000 (Invitrogen, Carlsbad, CA) following the instruction of the 123 manufacturer. The transfected cells were harvested at $48 \mathrm{~h}$ post-transfection for 124 subsequent analyses. Each experiment was repeated three times.

125 Vector construction, mutagenesis, and luciferase assay

126 The putative target genes of miR-16 were determined using online bioinformatics tools.

127 The results identified the presence of miR-16 binding sites in the 3' UTR of PAPP-A mRNA 128 and the promoter of UCA1, respectively. Subsequently, the 3' UTR of PAPP-A mRNA and 129 the promoter of UCA1 containing the miR-16 binding sites were amplified by PCR and 130 cloned into pcDNA vectors (Promega, Madison, WI) to generate wild type vectors for the 131 3' UTR of PAPP-A mRNA and the promoter of UCA1, respectively. At the same time, site132 directed mutagenesis was also carried out at the miR-16 binding sites in the 3' UTR of 133 PAPP-A mRNA and the promoter of UCA1, respectively. Subsequently, the mutant 134 sequences were also amplified by PCR and cloned into PcDNA vectors to generate mutant 135 vectors for the 3' UTR of PAPP-A mRNA and the promoter of UCA1, respectively. In the 136 next step, Trophoblast and Hela cells were co-transfected with UCA1 and miR-16, or miR13716 and PAPP-A mRNA, using Lipofectamine 2000. At $48 \mathrm{~h}$ after transfection, the 138 transfected cells were collected and the luciferase reporter activity in transfected cells was 139 detected by a Dual Luciferase Reporter Assay System (Promega, Madison, WI). The Renilla 140 internal control was used in the assay to normalize the relative firefly luciferase activity of 141 UCA1 and PAPP-A mRNA, respectively. Each experiment was repeated three times.

142 Western blot analysis 
143 Collected tissue and cell samples were lysed using a radioimmunoprecipitation assay 144 (RIPA) buffer (Invitrogen, Thermo Fisher Scientific, Waltham, MA) supplemented with 1\% 145 sodium deoxycholate, 1\% NP-40, 0.1\% SDS, $150 \mathrm{mM} \mathrm{NaCl}$ and $50 \mathrm{mM}$ Tris- $\mathrm{HCl}(\mathrm{pH} 8.8)$. 146 The lysis treatment was performed at $4^{\circ} \mathrm{C}$ for $30 \mathrm{~min}$ following the guideline provided by 147 the supplier. In the next step, the cell lysate was centrifuged at $4^{\circ} \mathrm{C}$ and $15,000 \mathrm{rpm}$ for $14810 \mathrm{~min}$. Subsequently, the bicinchoninic acid method was used to measure the 149 concentration of protein in the supernatant. Thereafter, $10 \%$ sodium dodecyl sulfate150 polyacrylamide gel electrophoresis (SDS-PAGE) was used to separate the protein in each 151 sample, and the separated proteins were then blotted onto a polyvinylidene difluoride 152 (PVDF) membrane (Immobilon-P; Millipore, Billerica, MA) for $60 \mathrm{~min}$ at $180 \mathrm{~mA}$ using a 153 transfer buffer containing 0.2 Mglycine, 20\% methanol, and $25 \mathrm{mM}$ Tris (Promega, 154 Madison, WI) following a standard protocol. Subsequently, the membrane was blocked in 155 5\% skim milk (Merck, Darmstadt, Germany) and then incubated with anti-PAPP-A (1:1000 156 dilution, Abcam, Cambridge, MA) and anti- $\beta$-actin primary antibodies (1:10,000 dilution, 157 Abcam, Cambridge, MA) overnight at $4^{\circ} \mathrm{C}$, followed by another 60 min of incubation with 158 horseradish peroxidase (HRP)-conjugated goat anti-rabbit immunoglobulin G secondary 159 antibodies (1:10,000 dilution, Abcam, Cambridge, MA). In the next step, the 160 chemiluminescent signals of bound antibodies were detected using an 161 Electrochemiluminescence Plus Western Blotting Detection System (GE Healthcare 162 Bio-Sciences, Pittsburgh, PA) in conjunction with a high-performance chemiluminescence 163 film (GE Healthcare Bio-Sciences, Pittsburgh, PA) in accordance with the manufacturer's 164 recommendation. The relative expression of PAPP-A protein in each sample was 165 normalized to the protein expression of the internal control $\beta$-actin. Each experiment was 166 repeated three times.

\section{Immunohistochemistry}

168 Sample sections were dewaxed twice in xylene (10 min each time) and dehydrated in 169 graded ethanol (100\%, 95\%, 80\%; and 70\%, 2 min each time). Subsequently, the sections 170 were placed on a shaker and washed twice in distilled water ( 5 min each time). In the next 171 step, the sections were incubated in $3 \% \mathrm{H}_{2} \mathrm{O}_{2}$ for 10 min and washed by distilled water, 
172 followed by antigen repair at high pressure for $90 \mathrm{~s}$. After being cooled down to room

173 temperature, the sections were washed with phosphate buffer saline (PBS), blocked with

$1745 \%$ bovine serum albumin (BSA) for $30 \mathrm{~min}$ at $37^{\circ} \mathrm{C}$, and then incubated overnight at $4^{\circ} \mathrm{C}$ 175 with anti-PAPP-A primary antibodies (1:100, Abcam, Cambridge, MA). After PBS washing, 176 the sections were further incubated with biotinylated secondary antibodies (1: 100, 177 Abcam, Cambridge, MA) at $37^{\circ} \mathrm{C}$ for $30 \mathrm{~min}$. Subsequently, after being fully washed, the 178 sections were incubated with horseradish peroxidase (HRP)-conjugated working fluid, 179 stained by 3,3-diaminobenzidine (DAB, chromogenic agent), and counterstained for $5 \mathrm{~min}$ 180 with hematoxylin. During this experiment, PBS was used as the negative control. After 181 staining, the cells showing positive expression of PAPP-A protein were analyzed under a 182 microscope. In brief, five fields of vision were chosen randomly from each section under 183 a high power lens to detect the localization of positive PAPP-A expression. Each 184 experiment was repeated three times.

185 ELISA

186 The level of PAPP-A protein expression in collected samples was measured using an ELISA 187 kit (Thermo Fisher Scientific, Waltham, MA) following the instruction of the kit. Each 188 experiment was repeated three times.

\section{Statistical analysis}

190 All statistical analysis was carried out using SPSS 19.0 software (SPSS, Chicago, IL). All data 191 were shown as mean \pm standard deviations (SD). The differences between two different groups were compared using Student's $t$-tests, while the differences between multiple groups were compared using one-way analysis of variance (ANOVA). The ROC curves were plotted using NCSS Statistical Software (NCSS, Kaysville, UT) to calculate the area under curve (AUC) values of different markers. At least three independent tests were performed for each experiment. A P value of $<0.05$ was considered statistically significant.

Results

199 In this study, demographic and clinical characteristics of all subjects were collected and 200 analyzed, as shown in Table 1. And there were no significant differences between 
201 different groups of PE patients as well as the case group with respect to the above indexes.

202 Moreover, as shown in Fig.1, upon the calculation of the diagnostic values of UCA1, miR-

20316 and PAPP-A in the diagnosis of PE, the AUC of miR-16 and PAPP-A was very similar, 204 while the AUC of UCA1 was evidently higher, suggesting that UCA1 can be used as a better 205 marker in the diagnosis of PE.

206 UCA1/PAPP-A expression was inhibited in PE cases along with an enhanced miR-16 207 expression

208 As shown in Fig.2A, PAPP-A expression was similarly down-regulated in the MILD group 209 and SEVERE group compared with that in the control group, which showed the highest 210 PAPP-A expression. On the contrary, the HELLP group showed the lowest PAPP-A 211 expression. Besides, the relative expression of UCA1 in the plasma (Fig.2C) showed the 212 same trend as that of log10 MoM PAPP-A. However, the relative expression of miR-16 in 213 the plasma (Fig.2B) showed the opposite trend: The expression of miR-16 was the highest 214 in the HELLP group and the lowest in the control group. Furthermore, the expression of 215 PAPP-A (Fig.3A), miR-16 (Fig.3B) and UCA1 (Fig.3C) in placenta samples collected from 216 different groups also showed a similar trend as that in the peripheral blood samples.

217 Finally, the trend in PAPP-A expression among different groups was also validated using 218 IHC assays (Fig.3D). Therefore, PE patients showed inhibited miR-16 expression and 219 increased UCA1/PAPP-A expression, while the patients with HELLP pregnancies showed 220 the lowest UCA1/PAPP-A expression and the highest miR-16 expression.

\section{UCA1 and PAPP-A mRNA functioned as a sponge and a target of miR-16, respectively}

222 Based on a computational analysis carried out using an online target prediction tool 223 (www.targetscan.org), a binding sequence of miR-16 was identified in UCA1 (Fig.4A). 224 Subsequently, a luciferase assay was performed to evaluate the interaction between miR22516 and UAC1, and the results demonstrated evidently reduced luciferase activity in 226 Trophoblast (Fig.4B) and Hela (Fig.4C) cells co-transfected with miR-16 and wild-type 227 UCA1, suggesting that UCA1 acts as a sponge of miR-16. Additionally, a 'seed sequence' 228 of miR-16 was identified in the 3'UTR of PAPP-A mRNA (Fig.4D), and the luciferase activity 
229 of Trophoblast (Fig.4E) and Hela (Fig.4F) cells was significantly reduced upon the co-

230 transfection with miR-16 and wild-type PAPP-A 3'UTR, indicating that PAPP-A mRNA acts

231 as a virtual target gene of miR-16.

\section{The establishment of the UCA1/miR-16/PAPP-A signaling pathway}

233 To further investigate the regulatory relationships among UCA1, miR-16, and PAPP-A, 234 Trophoblast cells were transfected with plasmids carrying UCA1 or anti-miR-16. As shown 235 in Fig.5, the relative expression of miR-16 (Fig.5A) was significantly suppressed in the 236 presence of UCA1 or anti-miR-16, along with significantly increased expression of PAPP-A 237 mRNA. Also, the results of Western blot analysis showed significantly increased 238 expression of PAPP-A protein (Fig.5C) in the UCA1 and anti-miR-16 groups, verifying the 239 role of UCA1 as a negative regulator of miR-16 expression. Unlike miR-16, a positive 240 relationship was confirmed between UCA1 and PAPP-A. Similar results were also obtained

241 from Hela cells (Fig.6), thus establishing a UCA1/miR-16/PAPP-A signaling pathway. In 242 summary, it can be concluded that when the expression of UCA1 is reduced, the 243 expression of PAPP-A is accordingly suppressed, thus exhibiting positive effects in the 244 pathogenesis of PE.

\section{Discussion}

246 As a major contributor to neonatal and maternal mortality and morbidity, PE is known for 247 its role as a trigger of maternal proteinuria, hypertension, imbalance of angiogenic factors, 248 and placental dysfunction [23]. During PE, the decrease in uteroplacental perfusion may 249 cause ischemia, which in turn promotes the release of soluble factors from the ischemic 250 placenta and subsequently causes hypertension and endothelial dysfunction [24]. The 251 physiological alterations observed in PE also promote the synthesis of proinflammatory 252 cytokines, including IL-6 and tumor necrosis factor-alpha (TNF-a), from the placenta [25]. 253 Recently, many studies showed that the dysregulation in the production of placental 254 growth factor (PIGF), vascular endothelial growth factor (VEGF), and soluble fms-like 255 tyrosine kinase I (sFlt-1) plays an essential role in the pathology of PE [26, 27]. 
256 UCA1 was first discovered in human bladder carcinoma [20]. Previous studies have 257 reported that UCA1 can promote the development of drug resistance, metastasis, and 258 proliferation in many types of cancer cells, including the cells of breast cancer and bladder 259 cancer [28-30]. UCA1 was recently found to function as a miRNA sponge. For example, 260 UCA1 can activate mTOR to regulate the expression of hexokinase 2 (HK2) and glycolysis 261 by activating STAT3 and by inhibiting the expression of miR-143 [31]. UCA1 also increases 262 the invasion and migration ability of epithelial ovarian cancer cells by directly binding to 263 miR-485-5p [32]. In this study, the AUC of miR-16 and PAPP-A was evidently lower than 264 that of UCA1, suggesting that UCA1 is associated with a better value in the diagnosis of 265 PE.

266 In both plasma and placenta samples collected from the subjects of this study, the 267 expression of PAPP-A and UCA1 was similarly down-regulated in the MILD and SEVERE 268 groups, while the patients in the HELLP group showed the lowest expression of PAPP-A 269 and UCA1. Meanwhile, the relative expression of miR-16 was the highest in the HELLP 270 group and the lowest in the control group, verifying the role of UCA1, miR-16 and PAPP271 A dysregulation in the pathogenesis of PE, especially in the pathogenesis of HELLP 272 pregnancies.

273 In addition, it was shown that miR-16 acts as a tumor suppressor by regulating the 274 apoptosis and proliferation of many types of cancer cells, such as bladder cancer cells [33]. 275 It was postulated that UCA1 can bind to miR-16 to impair its functions in bladder 276 cancer. Past results also indicated that UCA1 exerts a dose-dependent sponge effect on 277 miR-16. Besides, it was shown using luciferase assays that miR-16 can directly bind to the 2783 UTR of GLS2 mRNA, suggesting that UCA1 acts as a tumor suppressor and sponge of 279 miR-16 to block its role in promoting the tumorigenesis of bladder cancer. In this study, 280 miR-16 binding sites were identified on UCA1 and the 3'UTR of PAPP-A mRNA, 281 respectively. Also, the luciferase activity of cells was evidently reduced when the cells 282 were co-transfected with miR-16 and wild-type UCA1/PAPP-A mRNA. These results 283 suggested that UCA1 functions as a sponge of miR-16, while PAPP-A mRNA acts as a virtual 284 target gene of miR-16. In humans, miR-16 targets WNT3A, BCL2 and Cyclin D1 (CCND1), 
all of which can enhance cell cycle arrest at the $\mathrm{G} 1 / \mathrm{S}$ checkpoint and suppress the invasion, proliferation, and survival of cancer cells [34]. In fact, it has been shown that, upon DNA damage, the expression of miR-16 is quickly increased before returning to a normal value. Altered expression of miR-16 can dramatically change the activity of Wip1. For example, the silencing of miR-16 expression promoted the expression of Wip1, while the overexpression of miR-16 significantly inhibited the expression of Wip1 upon DNA damage. Some of these miR-16 potential targets like BCL2, Wip1, and CCND1 have been observed to be associated or involved in the pathogenesis of $\mathrm{PE}[35,36,37]$. An analysis carried out on the miRNA/miRNA GO network produced most connections for miR-16. Also, the overexpression of miR-16 suppressed the migration and proliferation of decidua derived mesenchymal stem cells (dMSCs) while inducing cell-cycle arrest by binding to CCNE1. Furthermore, the over-expression of miR-16 also decreased the angiogenesis potential of HUVECs and blocked the migration of trophoblast cells, and such effect of miR-16 may be due to its activity in inhibiting the production of VEGF-A by dMSCs. Interestingly, the expression of VEGF-A and CCNE1 is negatively related to the expression of miR-16 in dMSCs, suggesting that the change in miR-16 expression in dMSCs may be implicated in the pathogenesis of PE. In this study, compared with that in cells transfected with the negative control, the relative expression of miR-16 was suppressed in cells transfected with UCA1 or anti-miR-16. In addition, the expression of PAPP-A mRNA/protein was elevated in cells transfected with UCA1 or anti-miR-16. Therefore, in the signaling pathway of UCA1/miR-16/PAPP-A, UCA1 acted as a negative regulator of miR-16 expression and an enhancer of PAPP-A mRNA/protein expression.

As a protein with high concentrations in the circulation of pregnant women, PAPP-A was initially discovered in 1974, although its biological role remained unclear for several decades. Nevertheless, PAPP-A1 has been used clinically as a biomarker in the screening of Down's syndrome $[8,38]$. In the 1990s, it was reported that PAPP-A is associated with a protease activity against insulin-like growth factor binding protein-4 (IGFBP-4), and a unique feature of this protease activity is that the presence of IGF-II or IGF-I is required to cleave IGFBP-4 [39-41]. As a protein complex, PAPP-A plays an important role and its 
314 expression in PE patients is significantly decreased [22]. Therefore, PAPP-A may be used 315 as a potential biomarker for the early diagnosis of PE. Besides, when the serum level of 316 PAPP-A is used in conjunction with other serum biomarkers or imaging examinations, 317 including the ultrasound examination of the uterine artery in the first trimester [6, 7], it 318 may provide more valuable information for the early diagnosis of PE. Moreover, Canini et 319 al have shown that PAPP-A can be used as a more useful marker in the prediction of fetal 320 growth restriction than in the prediction of PE [42]. Spencer et al also showed that a low 321 level of serum PAPP-A in pregnant women is associated with an elevated risk of SGA, 322 regardless of the karyotype of the infants [43].

\section{Conclusion}

324 In summary, the findings of this study demonstrated that, compared with miR-16 and 325 PAPP-A, UCA1 can be used as a biomarker of higher diagnostic value in the diagnosis of 326 PE. Meanwhile, miR-16 was validated to act as a potential regulator of PAPP-A expression 327 while UCA1 was demonstrated to sponge the expression of miR-16. Therefore, the 328 signaling pathway of UCA1/miR-16/PAPP-A was established, in which the expression of 329 PAPP-A was positively regulated by UCA1. Consequently, the reduced expression of UCA1 330 could exert a positive effect by reducing the expression of PAPP-A in the pathogenesis of 331 PE.

\section{Conflict of interest}

333 None

334 Figure legends

335 Table 1

336 Demographic and clinical characteristics of subjects without PE ( $N=172)$, subjects with 337 mild PE $(\mathrm{N}=88)$, subjects with severe $\mathrm{PE}(\mathrm{N}=32)$ and subjects with HELLP pregnancies $338(\mathrm{~N}=8)$.

339 Figure 1. 
340 Diagnostic values of UCA1, miR-16 and PAPP-A were determined by calculating the AUC 341 of each index.

\section{$342 \quad$ Fig.2}

343 A. Log10 MoM PAPP-A in the plasma of MILD, SEVERE, HELLP and control groups;

344 B. Relative expression of miR-16 in the plasma of MILD, SEVERE, HELLP and control groups;

345 C. Relative expression of UCA1 in the plasma of MILD, SEVERE, HELLP and control groups.

\section{$346 \quad$ Fig.3}

347 A. Log10 MoM PAPP-A in the placenta samples of MILD, SEVERE, HELLP and control 348 groups;

349 B. Relative expression of miR-16 in the placenta samples of MILD, SEVERE, HELLP and 350 control groups;

351 C. Relative expression of UCA1 in the placenta samples of MILD, SEVERE, HELLP and 352 control groups;

353 D. Expression of PAPP-A protein in the placenta samples of MILD, SEVERE, HELLP and 354 control groups were detected using the IHC assay.

$355 \quad$ Fig.4

356 A. Computational analysis on the sequences of miR-16 and UCA1;

357 B. Luciferase assay of Trophoblast cells co-transfected with wild-type/mutant UCA1 and 358 miR-16/negative controls;

359 C. Luciferase assay of Hela cells co-transfected with wild-type/mutant UCA1 and miR360 16/negative controls;

361 D. Computational analysis on the sequences of miR-16 and PAPP-A mRNA;

362 E. Luciferase assay of Trophoblast cells co-transfected with wild-type/mutant PAPP-A 363 mRNA and miR-16/negative controls; 
364 F. Luciferase assay of Hela cells co-transfected with wild-type/mutant PAPP-A mRNA and 365 miR-16/negative controls.

\section{$366 \quad$ Fig.5}

367 A. Relative expression of miR-16 in Trophoblast cells transfected with negative controls, 368 UCA1, or anti-miR-16;

369 B. Relative expression of PAPP-A mRNA in Trophoblast cells transfected with negative 370 controls, UCA1, or anti-miR-16;

371 C. Relative expression of PAPP-A protein in Trophoblast cells transfected with negative 372 controls, UCA1, or anti-miR-16;

373 D. The relative density of the PAPP-A protein band in Trophoblast cells transfected with 374 negative controls, UCA1, or anti-miR-16.

\section{$375 \quad$ Fig.6}

376 A. Relative expression of miR-16 in Hela cells transfected with negative controls, UCA1, or 377 anti-miR-16;

378 B. Relative expression of PAPP-A mRNA in Hela cells transfected with negative controls, 379 UCA1, or anti-miR-16;

380 C. Relative expression of PAPP-A protein in Hela cells transfected with negative controls, 381 UCA1, or anti-miR-16;

382 D. The relative density of the PAPP-A protein band in Hela cells transfected with negative 383 controls, UCA1, or anti-miR-16.

\section{References}

3851 Sibai B, Dekker G, Kupferminc M: Pre-eclampsia. Lancet 2005;365:785-799.

3862 Moodley J: Maternal deaths due to hypertensive disorders in pregnancy. Best 387 Pract Res Clin Obstet Gynaecol 2008;22:559-567. 
3883 Wright A, Wright D, Syngelaki A, Georgantis A, Nicolaides KH: Two-stage screening 389 for preterm preeclampsia at 11-13 weeks' gestation. Am J Obstet Gynecol $390 \quad$ 2018;10.1016/j.ajog.2018.10.092

3914 Shennan AH: Recent developments in obstetrics. BMJ 2003;327:604-608.

3925 Bellamy L, Casas JP, Hingorani AD, Williams DJ: Pre-eclampsia and risk of 393 cardiovascular disease and cancer in later life: systematic review and meta394 analysis. BMJ 2007;335:974.

3956 Spencer K, et al.: First-Trimester Markers of Aneuploidy and the Prediction of Small396 for-Gestational Age Fetuses. Obstetrical \& Gynecological Survey 63.4(2008):215217.

3987 Spencer, Kevin: Screening for trisomy 21 in twin pregnancies in the first trimester 399 using free $\beta$-hCG and PAPP-A, combined with fetal nuchal translucency thickness. $400 \quad$ Prenat Diagn 20.2(2015):91-95.

4018 Lin TM, Galbert SP, Kiefer D, Spellacy WN, Gall S: Characterization of four human 402 pregnancy-associated plasma proteins. Am J Obstet Gynecol 1974;118:223-236. Kristensen T, Oxvig C, Sand O, Moller NP, Sottrup-Jensen L: Amino acid sequence of human pregnancy-associated plasma protein-A derived from cloned cDNA. Biochemistry 1994;33:1592-1598.

Stocker W, Grams F, Baumann U, Reinemer P, Gomis-Ruth FX, McKay DB, Bode W: The metzincins--topological and sequential relations between the astacins, adamalysins, serralysins, and matrixins (collagenases) define a superfamily of zincpeptidases. Protein Sci 1995;4:823-840.

1 Lawrence JB, Oxvig C, Overgaard MT, Sottrup-Jensen L, Gleich GJ, Hays LG, Yates JR, 3rd, Conover CA: The insulin-like growth factor (IGF)-dependent IGF binding protein-4 protease secreted by human fibroblasts is pregnancy-associated plasma protein-A. Proc Natl Acad Sci U S A 1999;96:3149-3153.

12 Pihl, Kasper, et al.: First trimester maternal serum PAPP-A, $\beta-$ hCG and ADAM12 in prediction of small-for-gestational-age fetuses. Prenatal Diagnosis 28.12(2008):1131-1135. 
$41713 \quad$ Kuc, Sylwia, et al.: Evaluation of 7 Serum Biomarkers and Uterine Artery Doppler Ultrasound for First-Trimester Prediction of Preeclampsia: A Systematic Review. Obstetrical \& Gynecological Survey 66.4(2011):225-239.

14 Meekins JW, et al.: A study of placental bed spiral arteries and trophoblast invasion

15 Vassart G and Dumont JE: The thyrotropin receptor and the regulation of thyrocyte function and growth. Endocr Rev 13: 596-611, 1992.

16 Marques AC and Ponting CP: Intergenic IncRNAs and the evolution of gene expression.

42717 Chen G, Yin K, Shi L, Fang Y, Qi Y, Li P, Luo J, He B, Liu M and Shi T: Comparative

18 Martens-Uzunova ES, Bottcher R, Croce CM, Jenster G, Visakorpi T, Calin GA: Long noncoding RNA in prostate, bladder, and kidney cancer. Eur Urol 2014;65:11401151. analysis of human protein-coding and noncoding RNAs between brain and 10 mixed cell lines by RNA-Seq. PLoS One 6: e28318, 2011.

19 Ribeiro AO, Schoof CR, Izzotti A, Pereira LV, Vasques LR: MicroRNAs: modulators of cell identity, and their applications in tissue engineering. Microrna 2014;3:4553.

20 Xiao Y, Jiao C, Lin Y, Chen M, Zhang J, Wang J, Zhang Z: IncRNA UCA1 Contributes to Imatinib Resistance by Acting as a ceRNA Against miR-16 in Chronic Myeloid Leukemia Cells. DNA Cell Biol 2017;36:18-25.

21 Li HJ, Li X, Pang H, Pan JJ, Xie XJ, Chen W: Long non-coding RNA UCA1 promotes glutamine metabolism by targeting miR-16 in human bladder cancer. Jpn J Clin Oncol 2015;45:1055-1063.

22 De Villiers CP, Hedley PL, Placing S, Wojdemann KR, Shalmi AC, Carlsen AL, Rode L, Sundberg K, Tabor A, Christiansen M: Placental protein-13 (PP13) in combination with PAPP-A and free leptin index ( $\mathrm{fLI}$ ) in first trimester maternal serum screening for severe and early preeclampsia. Clin Chem Lab Med 2017;56:65-74. 
44623 Duley L: The global impact of pre-eclampsia and eclampsia. Semin Perinatol $447 \quad 2009 ; 33: 130-137$.

44824 Spradley FT, Palei AC, Granger JP: Immune Mechanisms Linking Obesity and $449 \quad$ Preeclampsia. Biomolecules 2015;5:3142-3176.

45025 Shaw J, Tang Z, Schneider H, Salje K, Hansson SR, Guller S: Inflammatory processes 451 are specifically enhanced in endothelial cells by placental-derived TNF-alpha: 452 Implications in preeclampsia (PE). Placenta 2016;43:1-8.

45326 Herraiz I, Simon E, Gomez-Arriaga PI, Martinez-Moratalla JM, Garcia-Burguillo A, 454 Jimenez EA, Galindo A: Angiogenesis-Related Biomarkers (sFlt-1/PLGF) in the 455 Prediction and Diagnosis of Placental Dysfunction: An Approach for Clinical $456 \quad$ Integration. Int J Mol Sci 2015;16:19009-19026.

45727 Liu Z, Afink GB, Dijke PT: Soluble fms-like tyrosine kinase 1 and soluble endoglin 458 are elevated circulating anti-angiogenic factors in pre-eclampsia. Pregnancy $459 \quad$ Hypertens 2012;2:358-367.

46028 Wang F, Li X, Xie X, Zhao L, Chen W: UCA1, a non-protein-coding RNA up-regulated 461 in bladder carcinoma and embryo, influencing cell growth and promoting invasion. FEBS Lett 2008;582:1919-1927.

29 Huang J, Zhou N, Watabe K, Lu Z, Wu F, Xu M, Mo YY: Long non-coding RNA UCA1 promotes breast tumor growth by suppression of p27 (Kip1). Cell Death Dis 2014;5:e1008.

Fan Y, Shen B, Tan M, Mu X, Qin Y, Zhang F, Liu Y: Long non-coding RNA UCA1 increases chemoresistance of bladder cancer cells by regulating Wnt signaling. FEBS J 2014;281:1750-1758.

47232 Yang Y, Jiang Y, Wan Y, Zhang L, Qiu J, Zhou S, Cheng W: UCA1 functions as a 473 competing endogenous RNA to suppress epithelial ovarian cancer metastasis. Tumour Biol 2016;37:10633-10641. 
47533 Aqeilan RI, Calin GA, Croce CM: miR-15a and miR-16-1 in cancer: discovery, 476 function and future perspectives. Cell Death Differ 2010;17:215-220.

47734 Bonci D, Coppola V, Musumeci M, Addario A, Giuffrida R, Memeo L, D'Urso L, 478 Pagliuca A, Biffoni M, Labbaye C, Bartucci M, Muto G, Peschle C, De Maria R: The 479 miR-15a-miR-16-1 cluster controls prostate cancer by targeting multiple oncogenic activities. Nat Med 2008;14:1271-1277.

48135 Füsun Varol, et al.: VEGFR-1, Bcl-2, and HO-1 ratios in pregnant women with hypertension. Clinical and Applied Thrombosis/Hemostasis 21.3(2013).

483

36 Nuzzo AM, et al.: JunB/Cyclin-D1 imbalance in placental mesenchymal stromal cells 484 derived from preeclamptic pregnancies with fetal-placental compromise. Placenta 35.7(2014):483-490.

37 Tan B, et al.: The Regulation of Trophoblastic p53 Homeostasis by the p38-Wip1 Feedback Loop is Disturbed in Placentas from Pregnancies Complicated by Preeclampsia. Cell Physiol Biochem. 2019;52(2):315-335

41 Parker A, Gockerman A, Busby WH, Clemmons DR: Properties of an insulin-like growth factor-binding protein-4 protease that is secreted by smooth muscle cells.

50242 Canini S, Prefumo F, Pastorino D, Crocetti L, Afflitto CG, Venturini PL, De Biasio P: 503 Association between birth weight and first-trimester free beta-human chorionic 

2008;89:174-178.

50643 Spencer K, Cowans NJ, Avgidou K, Molina F, Nicolaides KH: First-trimester biochemical markers of aneuploidy and the prediction of small-for-gestational age fetuses. Ultrasound Obstet Gynecol 2008;31:15-19. 


\begin{tabular}{|c|c|c|c|c|c|}
\hline Characteristics & Control (N=172) & Mild PE (N=88) & Severe $P E(N=32)$ & HELLP (N=8) & $P$ value \\
\hline Age, years & $32.5 \pm 5.3$ & $33.1 \pm 6.2$ & $33.0 \pm 5.8$ & $31.5 \pm 4.8$ & ns \\
\hline BMI (kg/m2) & $21.5 \pm 5.1$ & $23.8 \pm 6.5$ & $24.4 \pm 5.2^{*}$ & $25.4 \pm 7.2^{*}$ & $<0.001$ \\
\hline Gravida & & & & & ns \\
\hline $1(\%)$ & $92(53.5)$ & $48(54.5)$ & $17(53.1)$ & $4(50.0)$ & \\
\hline $2(\%)$ & $45(26.2)$ & $24(27.3)$ & $9(28.1)$ & $2(25.0)$ & \\
\hline $3(\%)$ & $19(11.0)$ & 9 (10.2) & $3(9.4)$ & $1(12.5)$ & \\
\hline$>3(\%)$ & $16(9.3)$ & $7(8.0)$ & $3(9.4)$ & $1(12.5)$ & \\
\hline Birth weight, g & $3654.6 \pm 167.7$ & $3218.6 \pm 182.4^{*}$ & $3239.7 \pm 176.8^{*}$ & $3215.4 \pm 147.9 *$ & $<0.001$ \\
\hline GA at birth, days & $285.6 \pm 18.4$ & $269.1 \pm 17.9^{*}$ & $268.4 \pm 17.5^{*}$ & $272.4 \pm 21.5^{*}$ & $<0.001$ \\
\hline GA sampling, days & $92.6 \pm 8.4$ & $92.4 \pm 6.8$ & $91.9 \pm 10.4$ & $91.8 \pm 6.9$ & ns \\
\hline
\end{tabular}

Table 1. demographic and clinicopathological characteristics of the participants of this study $\left({ }^{*} \mathrm{P}<0.05\right.$ as compared with control) 


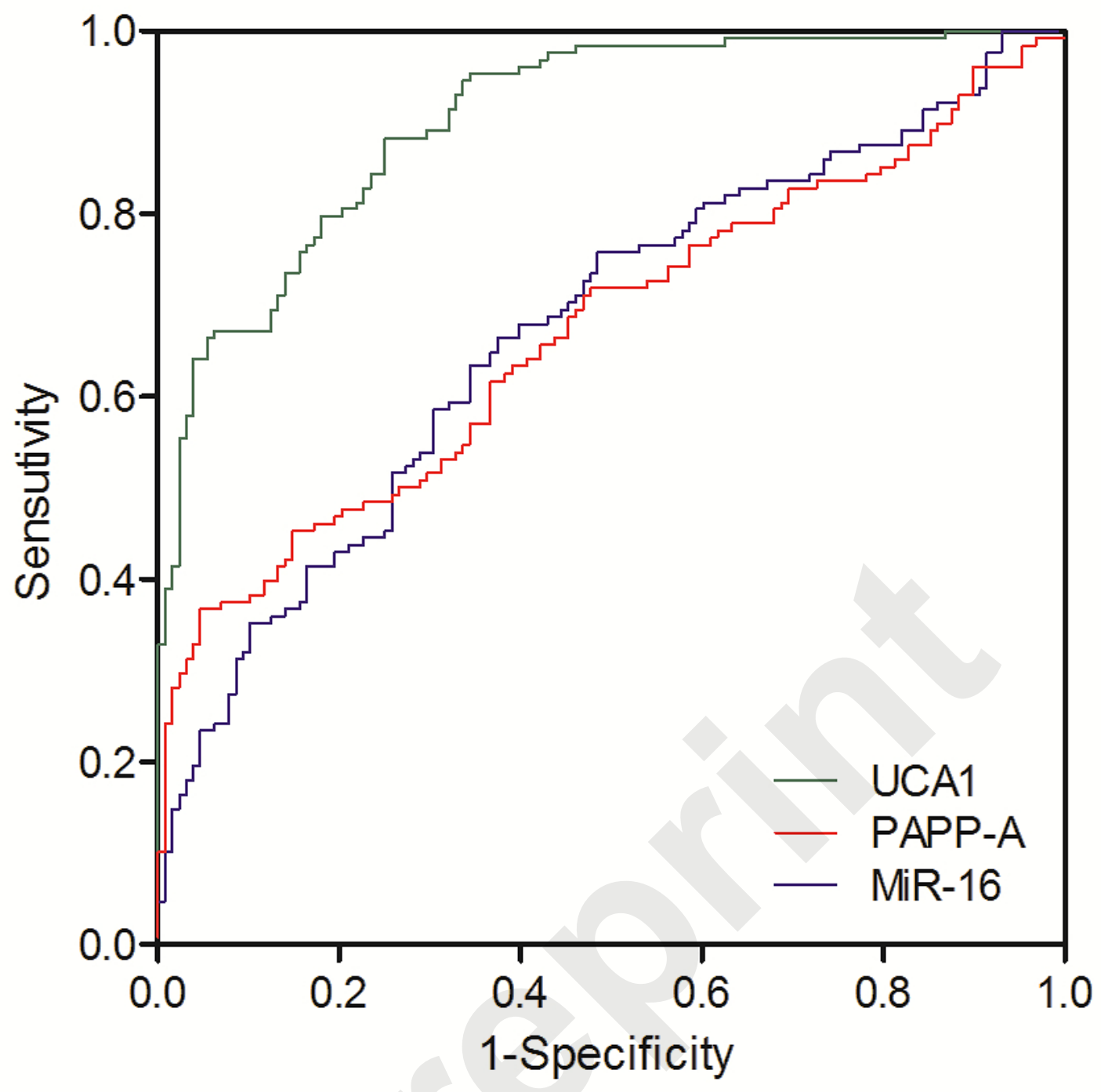




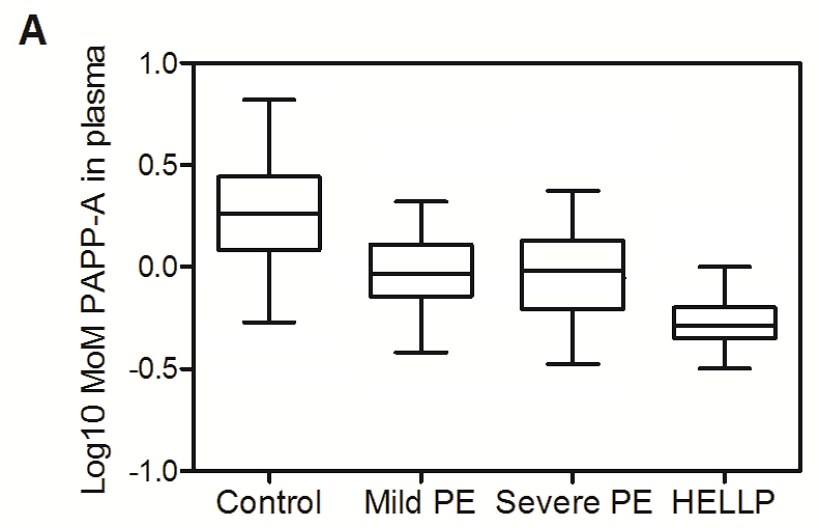

B

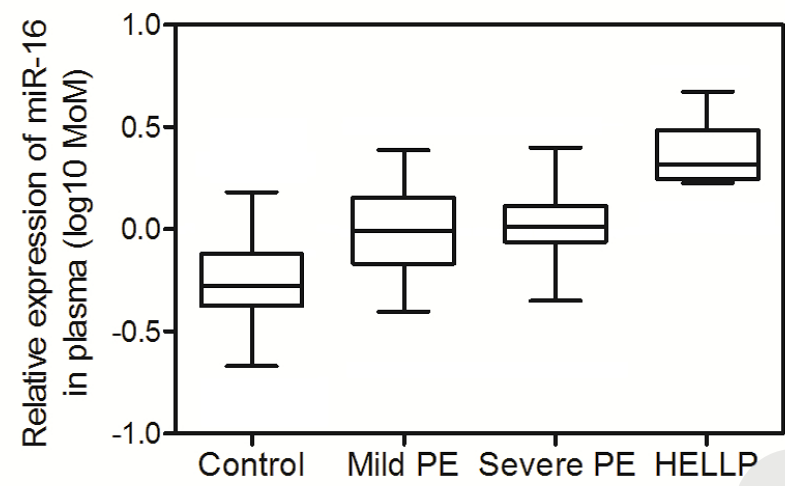

C

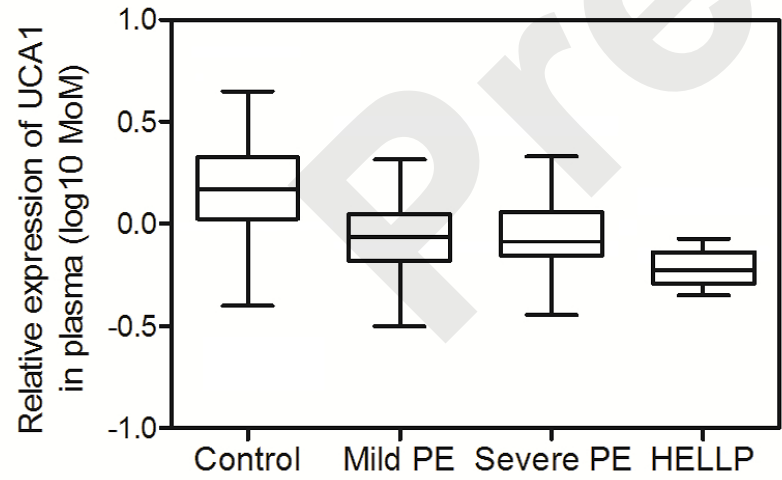


A

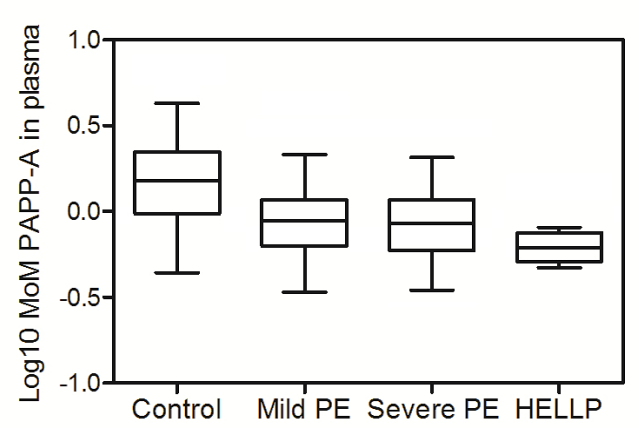

B

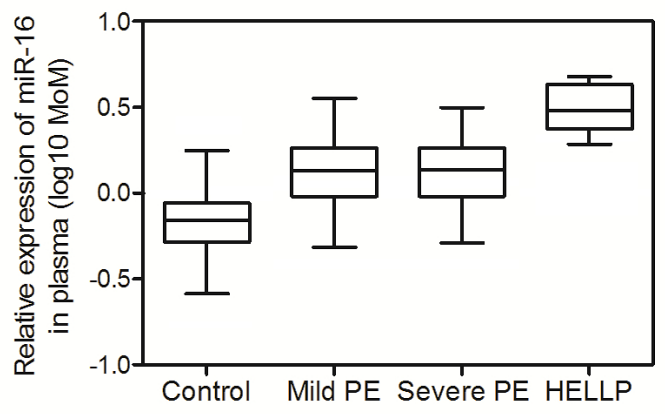

C

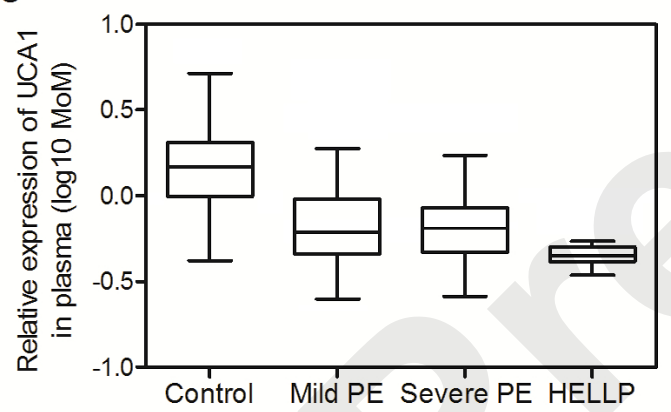

D Control Mild PE Severe PE HELLP

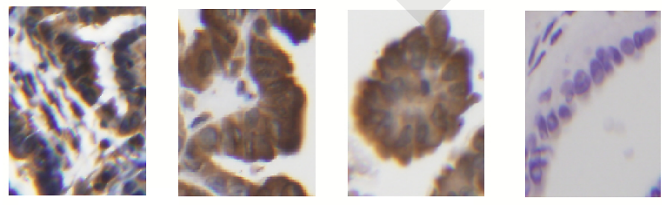


A

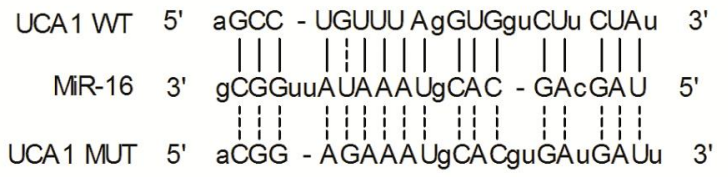

B

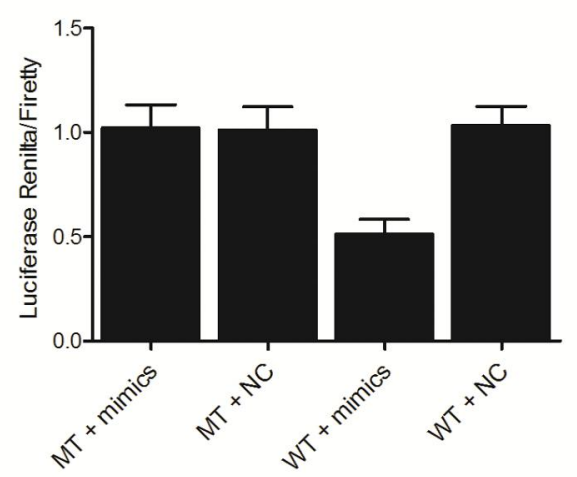

C

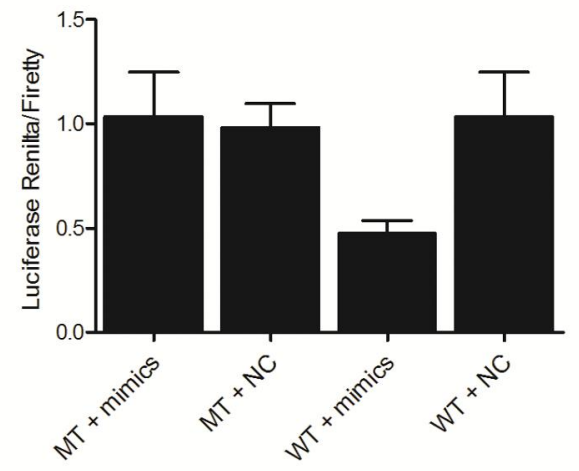

D

PAPPA WT 5' cT CGT CGTcctttcatcgtcgtt 3 ' MiR-16 3' uAGCAGCAcguaaauauugg $5^{\prime}$ Hinin

PAPPA MUT 5' cAGCAGCAcctttcatcgtcgtt $3^{\prime}$

E

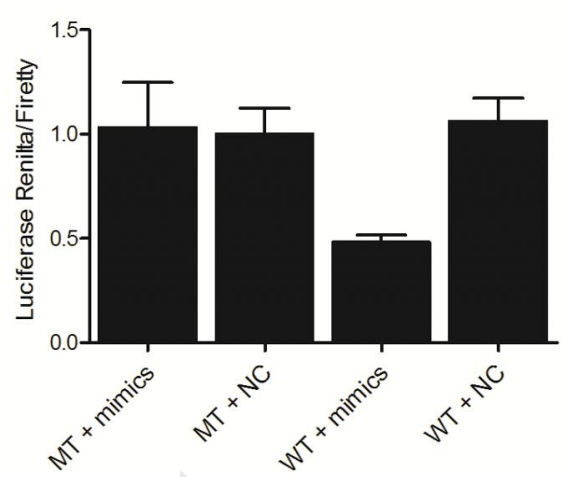

F

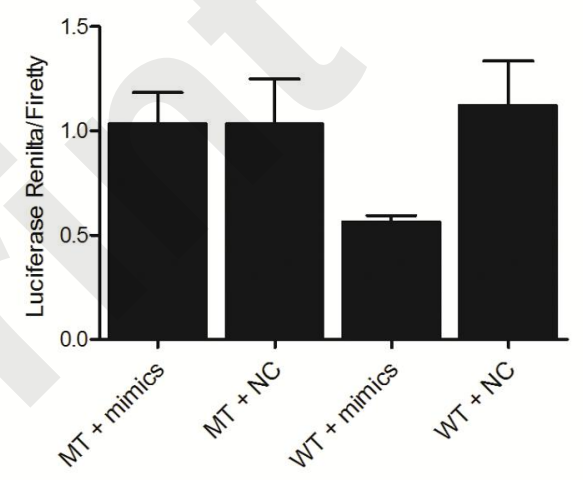



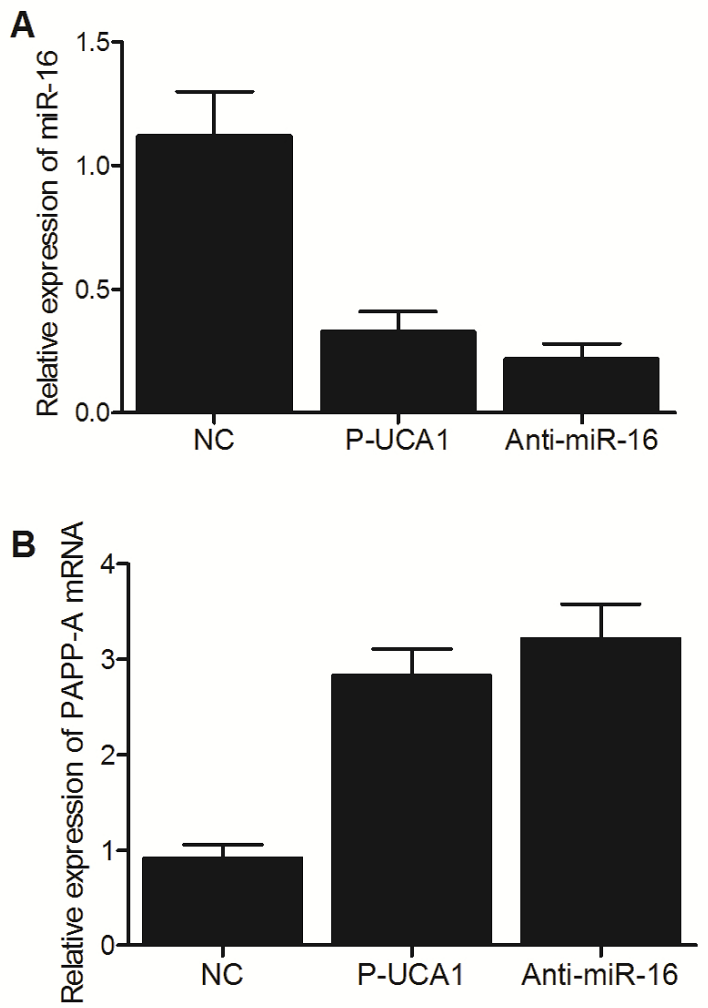

C
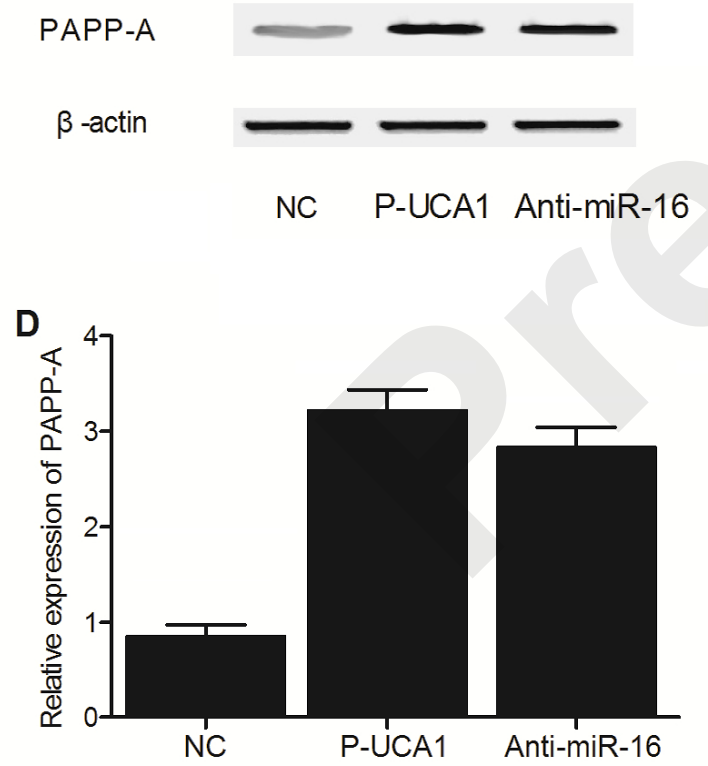

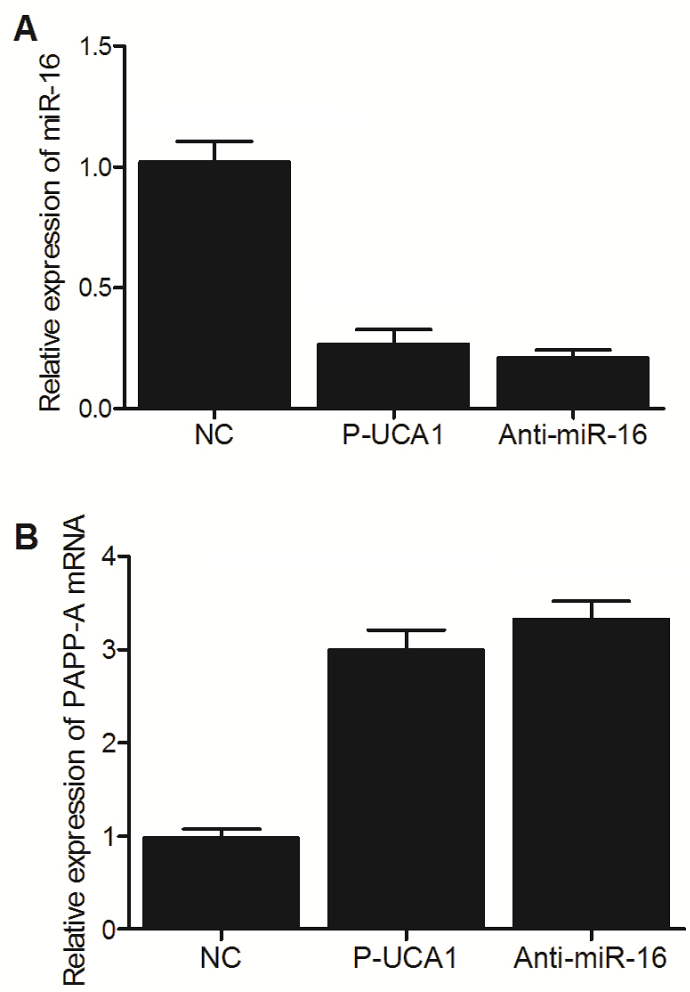

C
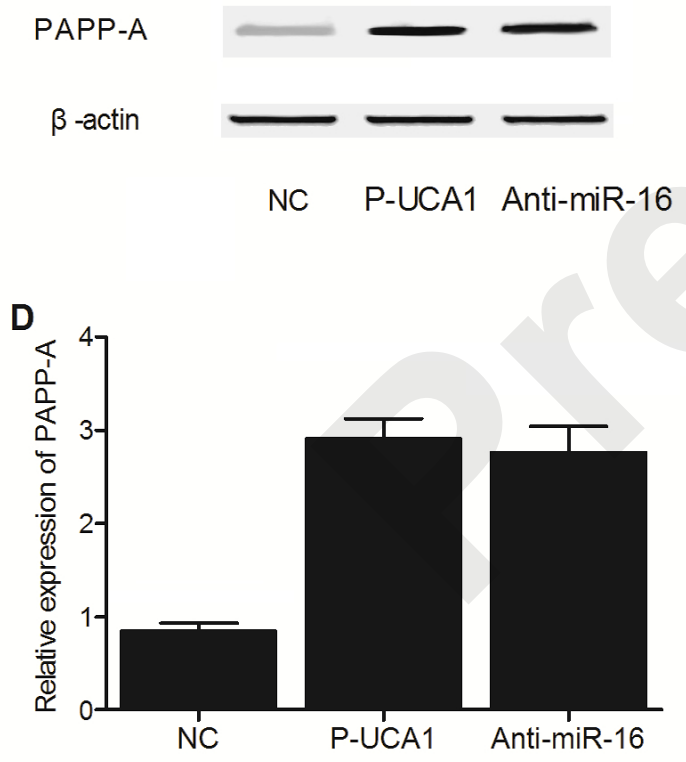